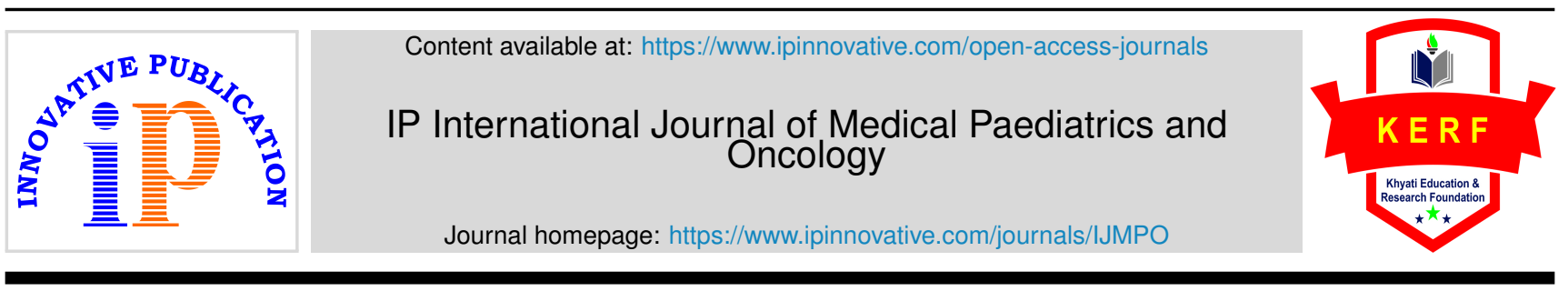

Original Research Article

\title{
A clinico pathological profile of dengue fever in children in urban areas of Telangana
}

\author{
Sri Krishna Surampud ${ }^{1}$, Kadukuntla Swapna Reddy ${ }^{1, *}$, Naila Mazher ${ }^{1}$, \\ Divya Sankuru ${ }^{1}$ \\ ${ }^{1}$ Dept. of Peadiatrics, Apollo Institute of Medical Sciences, Hyderabad, Telangana, India
}

\section{A R T I C L E I N F O}

\section{Article history:}

Received 27-07-2020

Accepted 31-10-2020

Available online 09-01-2021

\section{Keywords:}

Dengue virus infection

Fever

Thrombocytopenia

\begin{abstract}
A B S T R A C T
Background: Dengue is a common disease in this part of the world. It is one of the dreaded fevers for the paediatric age group. The disease has various presentations and features, but early diagnosis and management can decrease mortality rate significantly.

Aims: To evaluate the clinical profile of dengue infection in children under the age of 14 in urban areas of Telangana. The study is done in the tertiary care centre in urban area.

Materials and Methods: It is Hospital based descriptive, retrospective observational study. All probable cases of dengue were admitted with provisional diagnosis of dengue fever to the department of paediatrics for a period of one year. Total 198 children of age less than 14 years participated in present study.

Results:Out of 198 samples of clinically suspected dengue cases, 192 were found positive for Dengue NS1 antigen, 145 were found to be positive by IgG and 139 cases were positive for IgM. Majority of cases are males $(62 \%)$ females were $38 \%$. Most commonly affected age group is $6-10$ years of age 95 cases $(47.9 \%)$ Most of the patients in study are hospitalized for a period of 3-6 days (51.5\%). Fever (99.5\%) was the most common symptom found in all the patients followed by vomiting (17.7\%). Hypotension was found in $9 \%$ of the patients.

Severe thrombocytopenia was observed in $3.5 \%$ of patients and moderate thrombocytopenia in $7 \%$ of patients. $46.5 \%$ of patients had normal platelet count. Leukopenia was seen in $29.8 \%$ of patients.

Conclusions: Fever was the most common symptom found in all the patients. Majority of cases showed thrombocytopenia.

(C) This is an open access article distributed under the terms of the Creative Commons Attribution License (https://creativecommons.org/licenses/by/4.0/) which permits unrestricted use, distribution, and reproduction in any medium, provided the original author and source are credited.
\end{abstract}

\section{Introduction}

Dengue virus infection (DVI) is a mosquito-born disease and is a public health issue that is rising. The virus has been unwittingly transported from infectious areas to other parts of the world due to the upsurge of trade and tourist activity. ${ }^{1}$ The dramatic rise in dengue cases in Eastern India in 2012 became a public health issue as the majority of cases involved young teenagers. Infection with the Dengue virus causes substantial morbidity and mortality. India is one of the seven reported countries that routinely record dengue fever (DF) outbreaks in the South-East region,

\footnotetext{
* Corresponding author.

E-mail address: sapymbbs@gmail.com (K. S. Reddy).
}

and all four serotypes are known to circulate either alone or in combination. ${ }^{2}$ In terms of presentation, the clinical presentations range from moderate constitutional signs to extreme dengue hemorrhagic fever (DHF) and dengue shock syndrome (DSS) infections. While most infections are self-limiting, a small subset of patients experience serious complications that require intensive care. These complications arise very late in the illness, like organ loss, possibly offering a window of opportunity to identify a subset of patients who are likely to proceed through these complications.

It is difficult to manage dengue infections because it needs not only good control of the vectors responsible for viral spread, but also precise and rapid detection. To 
date, the precise and timely diagnosis of early detection of Dengue virus remains an issue in the management of patients with dengue infection in many parts of the world, especially in resource-limited countries. Warning signals "which may mean that patients may progress to a serious illness and need strict medical attention." Major plasma leakage, hemorrhagic complications, and/or serious organ dysfunction can manifest as severe dengue. The assumption that patient age affects the form and severity of symptoms is a concession on the sensitivity of the WHO classification system. Early case detection and treatment plays a key role in preventing both the seriousness and fatality of cases of dengue. The purpose of this research was to evaluate the clinical profile of dengue infection in children under the age of 14 in urban areas of Telangana.

\section{Materials and Methods}

It is Hospital based descriptive, prospective observational study. All probable cases with positive dengue tests were admitted with provisional diagnosis of dengue fever to the Paediatric Ward of Apollo Institute of Medical sciences from period June 2018 to May 2019 i.e. for a period of one year.

\subsection{Inclusion criteria}

All children aged up to 14 years with positive dengue tests, either NS1 antigen, IgM, IgG antibody rapid serological test kit or ELISA.

\subsection{Exclusion criteria}

Children who were positive for malaria, meningitis, and enteric fever.

The patients were exposed to all three serological examinations as the length of the history of fever may be fallacious. 198 were the total number of patients involved in our study. For the clinical and laboratory criteria, cases were followed up regularly. Blood parameters were tracked every day before remarkable clinical and haematological changes were seen. For each patient, averages of TLC, total platelet counts, $\mathrm{Hb}$, haematocrit, and so on were measured and reported. With the tourniquet test, daily vitals were registered. Chest $\mathrm{X}$-rays, ultrasonography, and liver function tests were conducted on all patients on day 3 of entry. As per the new WHO dengue guidelines, ${ }^{3}$ patients were treated with oral paracetamol, intravenous fluids and blood products, inotropes whenever necessary. The occurrence of different signs and symptoms was compared with the non severe and serious disease and the laboratory experiments. The observations have been tabulated and correlated.

The clinical manifestations and laboratory findings like haemoglobin estimation, total platelet count, haematocrit estimation, NS1 antigen, and IgM antibody of each group of illness were compared using Fisher's exact test for proportions. GraphPad version 6.0. software and SPSS version 22.0.0.0 software were used for data entry and analysis. value below 0.05 was considered significant.

\section{Results}

Total 198 children of age less than 14 years participated in present study.

Table 1: Results of samples tested by antigen NS1, IgG and IgM $(\mathrm{n}=198)$.

\begin{tabular}{|c|c|c|c|}
\hline $\begin{array}{l}\text { Results of the } \\
\text { test }\end{array}$ & NS1 & Ig G & Ig M \\
\hline Positive & $192(97 \%)$ & $145(73 \%)$ & $139(70 \%)$ \\
\hline Negative & $6(3 \%)$ & $53(27 \%)$ & $59(30 \%)$ \\
\hline
\end{tabular}

Out of 198 samples of clinically suspected dengue cases, 192 were found positive for Dengue NS1 antigen, 145 were found to be positive by IgG and IgM was positive for 139 cases.

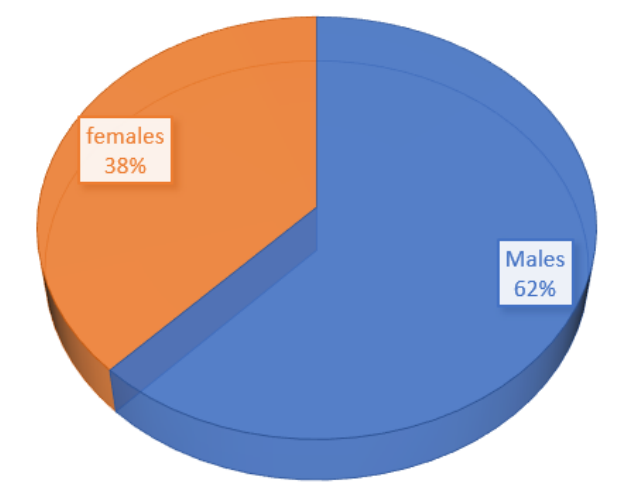

Majority of cases are males $62 \%$ and $38 \%$ were females

Fig. 1: Gender distribution in study

Table 2: Age distribution in study

\begin{tabular}{lcc}
\hline Age intervals & $\begin{array}{c}\text { Number of } \\
\text { patient } \mathbf{s}\end{array}$ & Percentages \\
<1 year & 12 & 6.1 \\
1-5 years & 36 & 18.2 \\
6-10 years & 95 & 47.9 \\
11-14 years & 55 & 27.8 \\
Total & 198 & 100 \\
Duration of & & \\
Hospitalization & & \\
<3 days & 20 & 10 \\
3-6 & 102 & 51.5 \\
$>6$ & 76 & 38.5 \\
\hline
\end{tabular}

Most commonly affected age group is 6- 10 years of age 95 cases $(47.9 \%)$ followed by $11-14$ years 55 cases $(27.8 \%)$, $1-5$ years 36 cases $(18.2 \%)$ and $<1$ year 12 cases $(6.1 \%)$. 
Most of the patients in study are hospitalized for a period of 3-6 days $(51.5 \%)$.

Table 3: Clinical signs and symptoms of dengue fever.

\begin{tabular}{lcc}
\hline Clinical features & $\begin{array}{c}\text { Number of } \\
\text { patients }\end{array}$ & Percentages \\
Fever & 197 & 99.5 \\
Vomiting & 35 & 17.7 \\
Abdominal pain & 7 & 3.5 \\
Rash & 6 & 3 \\
Loose stools & 5 & 2.5 \\
Cold & 5 & 2.5 \\
Head ache & 3 & 1.5 \\
Body pains & 4 & 2 \\
Bleeding manifestation & 2 & 1 \\
Hypotension & 18 & 9 \\
\hline
\end{tabular}

Fever $(99.5 \%)$ was the most common symptom found in all the patients followed by vomiting $(17.7 \%)$.

Table 4: Laboratory Parameters of Dengue Fever Patients.

\begin{tabular}{lcc}
\hline Parameters & $\begin{array}{c}\text { Number of } \\
\text { patients }\end{array}$ & Percentages \\
Total leucocyte count & 59 & 29.8 \\
$<4000 /$ cumm & 7 & \\
Platelet count <20,000 & 14 & 3.5 \\
20,000-50,000 & 92 & 7 \\
$50,000-1$ lakh & 49 & 46.5 \\
1 lakh-1.5 lakh & 36 & 24.7 \\
$>1.5$ lakh & & 18 \\
\hline
\end{tabular}

Significant changes in white blood cell counts and platelet counts were observed. Severe thrombocytopenia was observed in $3.5 \%$ of patients and moderate thrombocytopenia in $7 \%$ of patients. $46.5 \%$ of patients had mild thrombocytopenia. Leukopenia was seen in $25 \%$ of patients.

\section{Discussion}

Out of 198 samples of clinically suspected dengue cases, 192 were found positive for Dengue NS1 antigen, 145 were found to be positive by $\operatorname{IgG}$ and $\mathrm{IgM}$ was positive for 139 cases (Table 1). In study done by Chajhlana SPS et al. 137 samples of clinically suspected dengue cases, $119(86.9 \%)$ found to be positive dengue cases by laboratory confirmed cases (positive by one or more of the following tests NS1, antigen, IgM, IgG antibody). Study conducted by Anand et al found $83.3 \%$ positive cases. ${ }^{4,5}$

Majority of cases are males $62 \%$ and $38 \%$ were females (Figure 1). Chajhlana SPS et al and Tabassum et al in their study found that there was more number of males. also P P Vazhayil et al, K Jayasree et al and Shubhankar Mishra et al and Anand et alstudies. ${ }^{4,6-10}$

In the present study most commonly affected age group is 6- 10 years of age 95 cases (47.9\%)(Table-2). K Jayasree et al study shows 6-10 yrs. as most common affected age group similar to our study. Peter P Vazhayil et al study where maximum number of patients were in age above 11 years $(46 \%)$ which is contrast to our study. ${ }^{7,8}$

Most of the patients in study are hospitalized for a period of 3-6 days $(51.5 \%)$. In study done by Shubhankar Mishra et al. The mean duration of hospitalisation was 3.8 days with $63.9 \%$ of patients were admitted in the hospital for 3-6 days. ${ }^{9}$

In our study Fever (99.5\%)was the most common symptom found in all the patients followed by vomiting (17.7\%), (Table 3). In study done by Kumar SK et al. ${ }^{11}$ fever was present in most of the cases, followed by vomiting and abdominal pain which is also similar to the study conducted Ahmed $\mathrm{S}$ et al.in Pakistan. ${ }^{12}$ In the study conducted by Kashinkunti et al, ${ }^{13}$ Fever was the most common symptom found in all the patients followed by headache $(83.1 \%)$, Myalgia (77.3\%), retro- orbital pain (74.7\%). Hypotension was found in $86.5 \%$ of the patients. Kashinkunti et al, found the most common presentation was fever $100(100 \%)$, followed by headache $(90 \%)$, myalgia $(81 \%)$, vomiting $(56 \%)$ and abdominal pain $(48 \%) .^{13}$

In our study Severe thrombocytopenia was observed in $3.5 \%$ of patients and moderate thrombocytopenia in $7 \%$ of patients. $46.5 \%$ of patients had mild thrombocytopenia. Peter P Vazhayil, study where severe thrombocytopenia was seen in $8.97 \%$ cases and $\mathrm{K}$ Jayasree et al study where $(9.45 \%)$ had platelet counts. ${ }^{9}, 10$ Southeast Asian countries, report tourniquet test positivity as the commonest bleeding manifestation. Low proportion of positive tourniquet test in Indian studies may be due to the darker skin colour in Indian children. $^{14}$

Leukopenia was seen in $29.8 \%$ of patients which was similar to two other studies. ${ }^{14,15}$ The earliest haematological abnormality is a progressive decline in total WBC count in patients of dengue . Leukopenia was not significantly related with severe dengue cases which were against some results. ${ }^{16}$

The exact pathophysiology of thrombocytopenia in dengue is not yet clearly elucidated. Dengue virus may have a direct effect on the bone marrow -specially the progenitor cells causing a reduction in their capacity to replicate. An aberrant immunological response, implicated in severe dengue seems to play a significant role by dysregulation of plasma-kinin system. This leads to an increased consumption of platelets by disseminated intravascular coagulation (DIC). The damage is enhanced by increased apoptosis of platelets and generation of antiplatelet antibodies.

\section{Conclusion}

High grade fever, vomiting with normal or low platelet count were the presenting features in children. Early diagnosis, monitoring and prompt supportive management 
can reduce mortality.

Thrombocytopenia is one of the common findings . Dengue is a common acute febrile illness which comes as an epidemic in various parts of the country including Telangana. Over the recent years, it has emerged as one of the dreaded fevers in children. Knowledge and understanding of the varied presentations of DF in a region will definitely help in improving the outcome of this potentially fatal disease.

\section{Conflict of Interest}

None.

\section{Source of Funding}

None.

\section{References}

1. Wilder-Smith A. Dengue infections in travellers. Paediatr Int Child Health. 2012;32(1):28-32. 10i:10/179/20469047/2z00000000050.

2. Gubler DJ. Dengue and Dengue Hemorrhagic Fever. Clin Microbiol

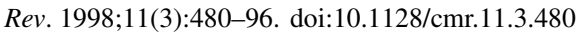

3. Hadinegoro SR. The revised WHO dengue case classification: does the system need to be modified? Paediatr Int Child Health.

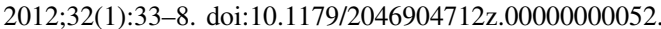

4. Chajhlana SPS, Mahabhasyam RN, Varaprasada MSM, Anukolu RSR. Socio demographic and clinical profile of dengue fever cases at a tertiary care hospital, Hyderabad, Telangana. Int J Community Med Public Health . 2017;4(6):2027-30. 1010.18203/2304$6040.11 \mathrm{cmph} 20172 \mathrm{~T} 70$

5. Anand AM, Sistla S, Dhodapkar R, Hamide A, Biswal N, Badrinathsrinivasan. Evaluation of NS1 Antigen Detection for Early Diagnosis of Dengue in a Tertiary Hospital in Southern India. J Clin Diagn Res. 2016;10(4):1-4.

6. Begum TM, Sumana MN, Gowdappa BH. Evaluation of Rapid ICT in comparison with MAC- ELISA in diagnosis of dengue fever at a tertiary care hospital, South India. Int J Pharm Sci Invention. 2014;3(12):11-6.

7. Vazhayil PP, Stephen ST, Kumar V. A retrospective observational study of dengue fever in a tertiary care center in Kerala. Int J Scientific Stud. 2017;5(1):30-4.
8. Jayashree K, Manasa GC, Pallavi P, Manjunath GV. Evaluation of Platelets as Predictive Parameters in Dengue Fever. Indian J Hematol

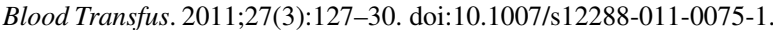

9. Mishra S, Ramanathan R, Agarwalla SK. Clinical Profile of Dengue Fever in Children: A Study from Southern Odisha, India. Scientifica (Cairo) . 2016;2016:1-6. 101:0/155/2016/6391594.

10. Anand KSS, Bettegowda S. Clinical and laboratory pattern of dengue fever: a retrospective study from rural hospital. Galore Int $J$ Health Sci Res. 2019;4(3):44-6.

11. Kumar SK, Rajendran NK, Brabhukumar AC. Clinical profile of dengue fever in children: analysis of 2017 outbreak from Central Kerala, India. Int J Contemp Pediatr. 2018;5(6):2265-9. 01:10.18203/2349-3291.1]cp20184293.

12. Ahmed S, Arif F, Yahya Y. Dengue fever outbreak in Karachi 2006- a study of profile and outcome of children under 15 years of age. $J$ Pak Med Assoc. 2008;58(1):4-8.

13. Kashinkunti M, Shiddappa, Dhananjaya MA. Study of Clinical Profile of Dengue Fever in a Tertiary Care Teaching Hospital. Sch J App Med Sci. 2013;1(4):280-2.

14. Seneviratne SL, Malavige GN, de Silva HJ. Pathogenesis of liver involvement during dengue viral infections. Trans Royal Soc Trop Med Hygiene. 2006;100(7):608-14. do1:10.1016/1.trstmh.2005.10.007

15. Mittal H, Faridi MMA, Arora SK, Patil R. Clinicohematological Profile and Platelet Trends in Children with Dengue During 2010 Epidemic in North India. Indian J Pediatr. 2012;79(4):467-71. 60i:10.1007/s $2008-011-0586-7$

16. Kularatne SA. Dengue fever. BMJ. 2015;351:h4661. do1:10.1136/bm..h466.

\section{Author biography}

Sri Krishna Surampud, Associate Professor

Kadukuntla Swapna Reddy, Assistant Professor

Naila Mazher, Assistant Professor

Divya Sankuru, Senior Resident

Cite this article: Surampud SK, Reddy KS, Mazher N, Sankuru D. A clinico pathological profile of dengue fever in children in urban areas of Telangana. IP Int J Med Paediatr Oncol 2020;6(4):143-146. 